\title{
A New Type of Micromanipulator and Microforge
}

\author{
By V. B. D. SKERMAN \\ Department of Microbiology, University of Queensland, \\ Medical School, Brisbane, Australia
}

(Accepted for publication I I July I968)

\section{INTRODUCTION}

The need for instruments which can be used for the manipulation of microscopically small objects has led to the invention of several micromanipulators. The more refined of these are research tools, the high cost of which matches their precision and mitigates against their general use, particularly by undergraduate students. With more attention being paid to genetics and the study of microcolony development in bacteria and unicellular algae, a greater interest has developed in the separation of single cells from microcolonies and in the selection of microcolonies themselves to prevent overgrowth by a more dominant population. This has increased the need for a simple form of micromanipulator and microforge. The author possesses the hydraulically operated de Fonbrun micromanipulator and microforge and also a Zeiss Jena micromanipulator. Both of these are excellent instruments but their design, apart from cost, makes difficult the handling of tools within the confines of the normal Petri dish. A new type of micromanipulator and microforge has therefore been designed which makes use of the optical systems normally available in the modern laboratory. Patent rights have been applied for in most leading countries.

\section{THE MICROMANIPULATOR}

This instrument was designed for use with the Reichert low-power $(\times 10)$ objective but can be used with suitable modification with any low-power objective with a working distance of about $7 \mathrm{~mm}$. or greater. It can be used with a normal light-field objective but a phase-contrast objective is to be preferred. It consists of two parts. One, for convenience, is called the lens collar (Fig. I) and the other the magnetic toolcarrier (Fig. 2). The assembled micromanipulator is shown in section in Fig. 3.

\section{The lens collar}

This consists simply of a non-ferrous metal (preferably brass) collar $A$ with a centre hole $B$ machined to fit closely but not too tightly to the outer casing of the low-power objective and secured to the objective casing with a non-metal screw $C$. A plastic liner (not shown) improves the fit. Attached to the side of the collar are two steel slides $D$ machined parallel and perfectly flat on the under surface. These slides are spaced equally on either side of the centre axis of the collar on the side opposite the retaining screw $C$. The width of the slides is determined by the width of the small magnet $H$ (Fig. 2) and the tool-carrying tube $J$. The inter-slide spacing $E$ should be not less than 3 times the outer diameter of the tool-carrying tube $J$, to permit adequate 


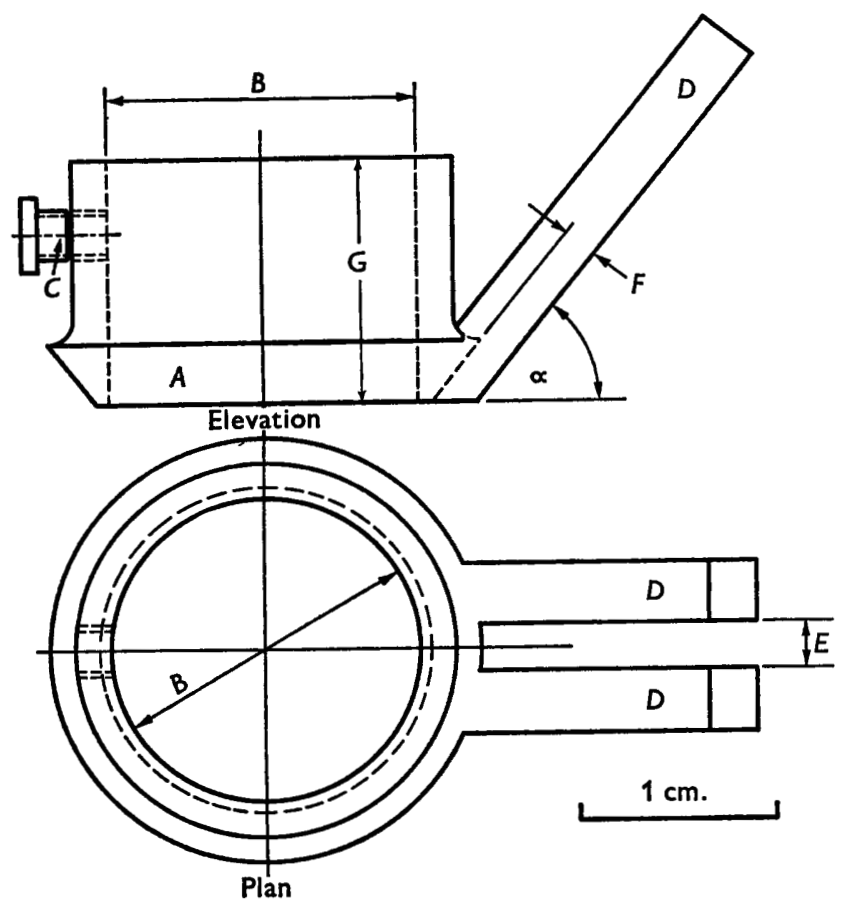

Fig. I. Lens collar. For explanation see text.

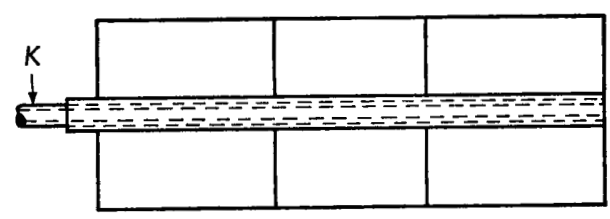

View underside
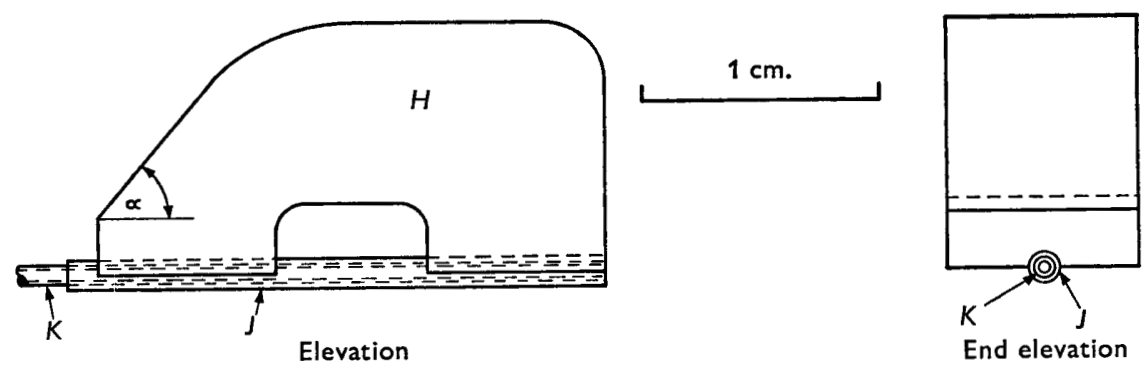

Fig. 2. Magnetic tool-carrier. For explanation see text. 
lateral movement in positioning the tool. The distance between the outer edges should not be less than the width of the magnet and preferably equal to it.

The thickness of the steel slides and their mode of attachment must be such that the clearance between the under surface of the slides and the metal collar (dimension $F$ ) is at least equal to the diameter of the tool-carrying tube $J$. This permits the magnet to slide freely along the steel slides without the tool-carrying tube $J$ fouling the lower edge of the collar. The angle of attachment of the steel slides must be such that when the magnet is in position the projected uppermost edge of the attached tool-carrying tube $J$ does not contact any of the metal parts of the objective when the projected centre axis of the tool-carrying tube $J$ is made to pass through the focal point of the front objective lens. For ideal operation the clearance should be at least $\mathrm{I} \cdot 5 \mathrm{~mm}$.

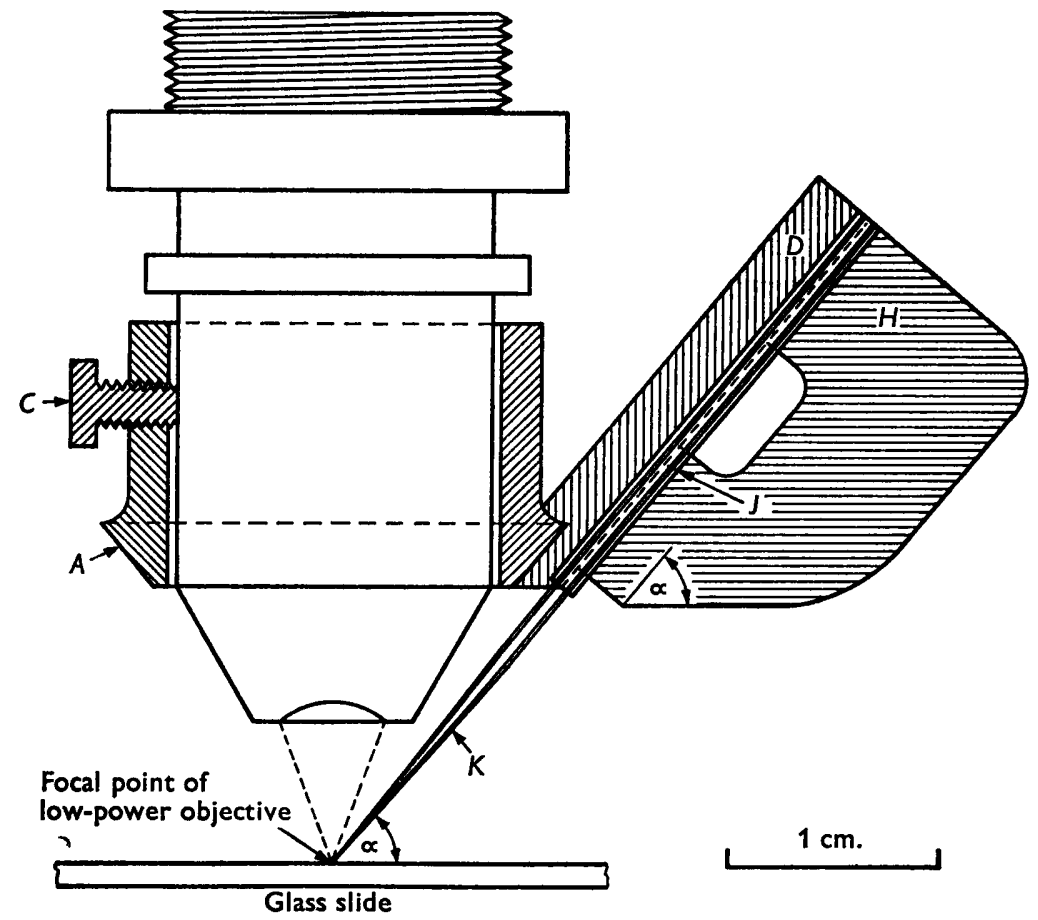

Fig. 3. Schematic drawing of micromanipulator in position. For explanation see text.

The height $G$ of the metal collar $A$ should be such as to provide a secure attachment of the lens casing and to allow vertical movement of the (projected) centre axis of the tool-holder at least I mm. above and below the focal point of the objective. The thickness of the collar should be adequate to provide a secure attachment for the screw $C$. If not, a metal extension should be provided for the attachment of the screw.

\section{The magnet tool-carrier}

The magnet $H$ may be of any dimensions but preferably should not exceed $25 \mathrm{~mm}$. in length or $10 \mathrm{~mm}$. in width or $12.5 \mathrm{~mm}$. in depth. The depth of the magnet should be sufficient to ensure that it can be easily gripped for manipulation between the thumb and index finger. An Eclipse Minor Magnet (catalogue no. 80I) manufactured 
by James Neill and Co. (Sheffield) Ltd., England, has proved very satisfactory. When a magnet of this type is used one end should be truncated at the angle $\alpha$ to ensure that its lowest point, when in the operative position, does not project near or below the focal point of the objective.

The tool-carrying tube $J$ is a cylindrical tube of any diameter but preferably not exceeding I mm. outer diameter and constructed of stainless steel. A section of a 19-gauge hypodermic needle has been used by the author, and it should exceed the length of the magnet by about $\mathrm{I} \mathrm{mm}$. It is recessed into the face of the magnet along the centre line and cemented to it with some material which will permit replacement of the tubing when required. The depth of the recess is not important, but when glass tools are to be used and are to be fabricated on the microforge the depth of the recess should be such that the centre axis of the tool-carrying tube $J$, when cemented into position, lies in the same plane as the face of the magnet.

The tool $K$ may be of glass or other material. For some purposes platinum wire, of the maximum gauge which will fit into the tool-carrying tube $J$, may be used and secured into the tool-holder with some malleable wax. The length of the extruded portion of the wire should be approximately equal to the distance between the lower end of the steel slides and the focal point of the objective when the tool is in the operating position. The tip of the platinum wire should be ground to a point on a fine honing-stone.

When glass tools are to be used good-quality soda-glass (preferably, since Pyrex is too brittle) tubing or rod should be drawn out to a diameter slightly smaller than the internal diameter of the tool-holder. A piece of this drawn tubing or rod is secured into the tool-holder with paraffin wax and severed from the end of the tool-carrying tube $J$ at a distance approximately equal to the distance between the lower end of the steel slides and the focal point of the lens when the tool is in its working position. The glass can be worked into any desired form with the microforge (Fig. 4).

\section{Operation}

The micromanipulator, without the magnetic tool-holder $H+J$, is secured to the low-power objective in the lowest position compatible with stability. The magnetic tool-holder $H J$, complete with tool $K$, is then attached to the steel slides and pushed down along the slides $D$ until the tip of the tool passes slightly beyond the centre of the lens. While viewing through the microscope, collar $A$ is slowly raised on the lens casing until the image of any part of the tool is brought to focus in the centre of the field. (It may be necessary to move the magnet slightly from side to side to align the tool-hence the gap between the steel slides.) The magnet is then drawn back up the steel slides until the tip of the tool $K$ is in the centre, when a final adjustment of the collar may be necessary. The magnet is then withdrawn further up the slide while a search is made for the specimen to be manipulated. Since the primary purpose in the design of the manipulator was the selection of single organisms or groups of organisms from microcolonies of bacteria, the colony is brought into focus in the centre of the field. The barrel of the microscope is then raised and the magnet pushed down till the tip of the tool returns to focus in the centre of the field. The microscope is then refocused on the colony. When this is done the tip of the tool makes contact with the centre of the colony. By re-positioning the specimen just before the colony comes into precise focus it is possible to manipulate from any desired area. 
The precise mode of manipulation depends on the design of the tool, which may be of a great variety of shapes. Three simple tools are the sharp probe, the knob, and the microloop. Of these the knob and the loop are the most useful for singleorganism isolations, the probe for marking the position of an organism on the agar surface.

Single-organism isolation by probe or loop, from a butyrous type colony, can be achieved simply by lowering the tool onto the edge of the colony, lifting it free from the agar, moving the Petri dish with the mechanical stage (modified to grip the perimeter of the dish), followed by lowering the tool again onto the uninoculated agar surface. A succession of such motions will rapidly thin out the population, finally resulting in the deposition of one organism or a small group of organisms.

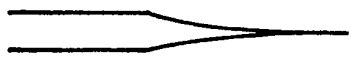

(a)

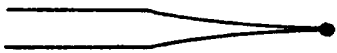

(b)

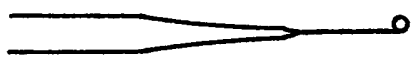

(c)

Fig. 4. Three simple tools: (a) sharp probe, $(b)$ knob, (c) microloop.
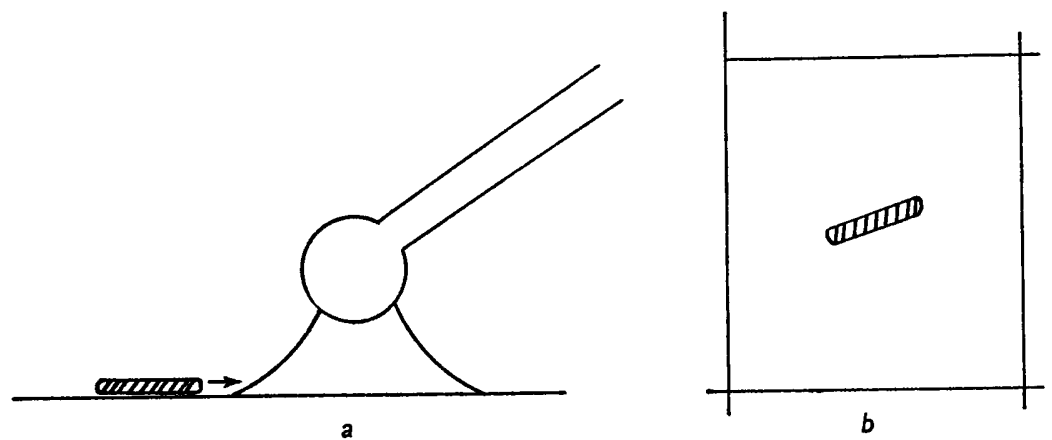

Fig. 5. $a$ Microtool pressing on agar. $b$ Position of organism marked by scoring surface of agar with probe.

At this stage the microtool should be replaced by a sterile tool, preferably a knob. When this is pressed down on the agar to one side of an organism, liquid will be expressed from the agar and the organism can be encouraged to float into it (Fig. $5 a$ ). Once this has occurred careful lateral movement of the Petri dish will permit the single organism to be floated across the agar, well clear of the original population. The position of this organism can be marked by scoring the surface of the agar with the probe (Fig. 5 b). After incubation the developing colony can be transplanted. Alternatively, the small block of agar supporting the organism can be excised under a stereo-microscope by using a sterile scalpel.

Attempts to lift single organisms in a loop for transfer to a new Petri dish are rarely successful, because of the rapid drying-up of the fluid. Transfer of masses of organisms in this manner can usually be effected. 


\section{THE MICROFORGE}

The manufacture of precise glass tools for use with the micromanipulator necessitates the use of some tool-fabricating device, of which several are marketed. The microforge described here makes use of the principle used in the de Fonbrun microforge and only one portion of the assembly here described (iii) is claimed to be original. The assembly can be used on any microscope fitted with a mechanical stage. It consists essentially of three parts.

$A$ regulated power supply. For this purpose the following electrical circuit shown in Fig. 6 is used.

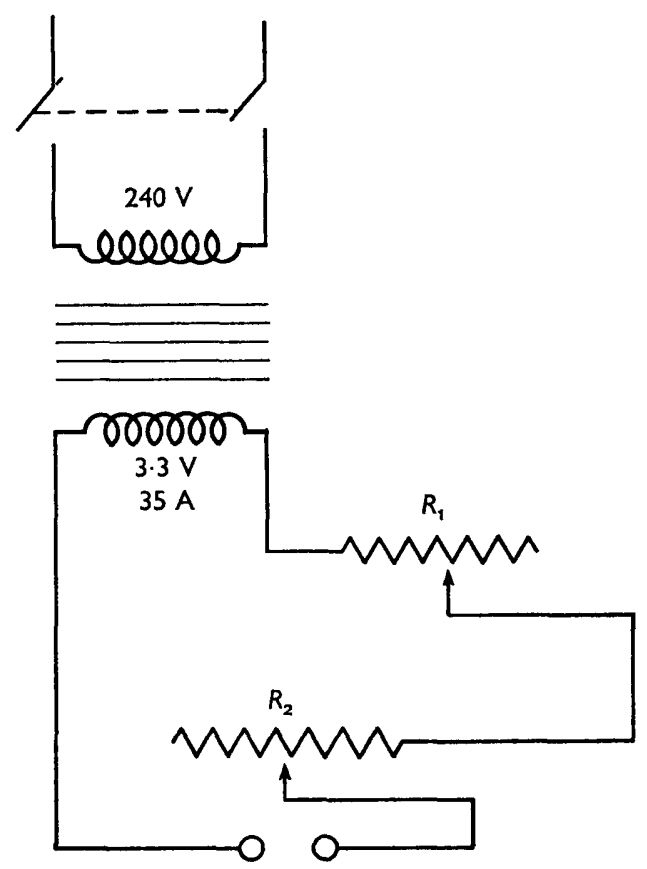

Fig. 6. Circuit for regulating power supply.

The transformer used is one manufactured by National Transformers Pty. Ltd., Australia (primary $240 \mathrm{~V} 50$ cycles; secondary $3.3 \mathrm{~V} ; 30 \mathrm{~A}$ intermittent rating). The variable $(7.07 \mathrm{~A})$ resistance $R_{1}$ is a $I \Omega$ Type $\mathrm{L}_{50} \mathrm{CF}$ manufactured by the British Electric Resistance Co. Ltd., Enfield, Middlesex, England. Variable resistance $\boldsymbol{R}_{\mathbf{2}}$ is a $2 \Omega$ Type $L_{100}\left(7^{\circ} 07 \mathrm{~A}\right)$. The sole purpose of resistance $R_{1}$ is to limit the maximum voltage across the element $L$ (Fig. 7 ; a $15 \mathrm{~mm}$. length of platinum/iridium wire) to a value which will not fuse the wire. Control of heating within this range is obtained with the variable resistance $R_{2}$.

The platinum/iridium heating stage (Fig. 7). The design of the holders $M$ for the platinum/iridium wire element $L$ is essentially the same as that of the de Fonbrun microforge. They are mounted in an insulated block $N$ on a metal slide $O$ and operated on an ordinary microscope by means of a mechanical stage. The height of the element 
$L$ above the slide $O$ must be greater than the distance between the centre of the hole $X$ and the base of the vertical support $V$ in Fig. 8 and 9 .

Microforge tool-carrier. The assembled microforge is shown in sectional diagram in Fig. 8 and in exploded form in Fig. 9. The tool-carrier consists essentially of the three parts described below.

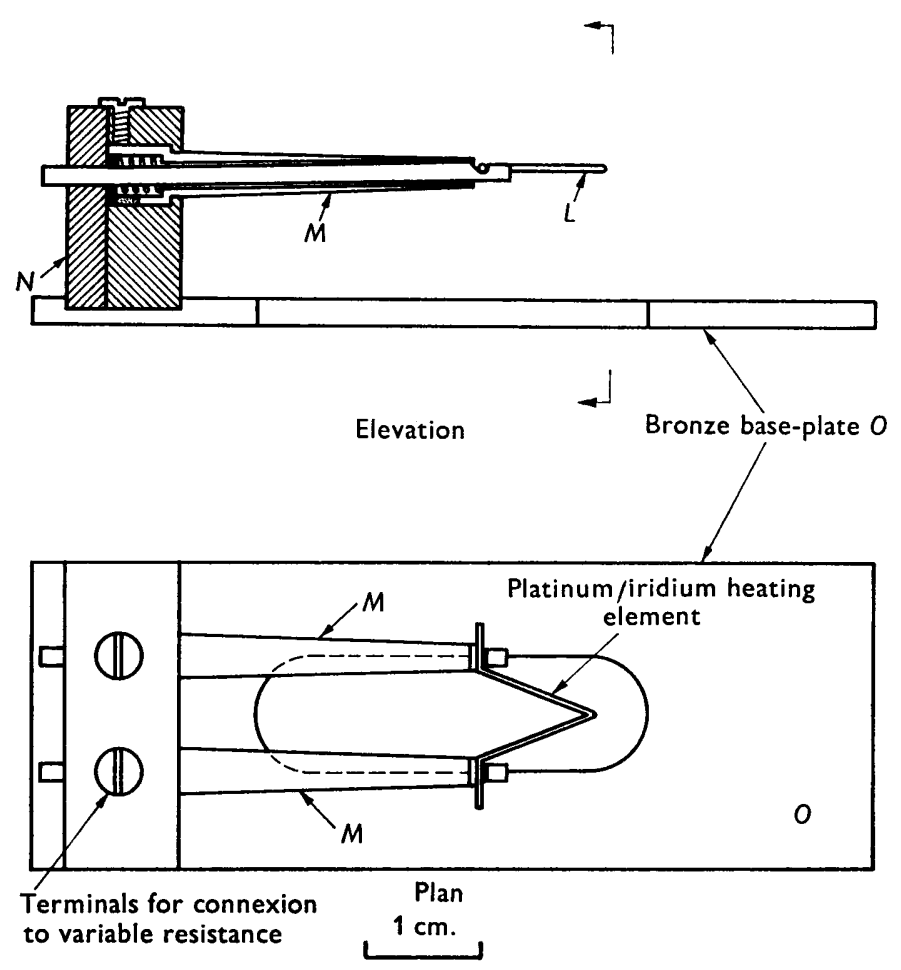

Fig. 7. Platinum/iridium heating stage. For explanation see text.

(i) A collar $P$ whose internal diameter is determined by the external diameter of the casing of the $\times 2.5$ objective lens $Q$ to which it is secured by a plastic screw $S$. The thickness of the wall must be such as to provide a secure thread base for the plastic screw. At the base of the collar is a flange $T$ upon which the ring $U$ rotates. Vertical adjustment of the position of the collar $P$ on the casing of the lens permits the glass tool $K$ to be brought to focus at the focal point of the objective lens when the ring $U$ is seated on the flange $T$.

(ii) A ring $U$ to which is attached the vertical support $V$. The internal diameter of the ring should be such that it fits closely but not too tightly to the collar $P$. The thickness of the ring should be such as to permit the fixing of the vertical support $V$ and to separate it from the flange $T$ at the base of the collar $P$ by about I mm. The height of the ring $U$ should be sufficient to ensure a rock-free fit on the collar and adequate space for the insertion of the plastic screws $W_{I}$ and $W_{2}$ in the ring. The plastic screws in the ring should (for convenience only) be located opposite to and at right angles to the vertical support. 
A hole $X, 5 \mathrm{~mm}$. in diameter, is drilled through the brass supporting plate $V$ which is positioned on $U$ so that the centre of the hole lies on a line in the same plane as the centre of the ring $U$. The plate $V$ is milled on its outer face to a depth sufficient to receive a polytetrafluoroethylene (PTFE) washer and the circular plate $Y_{1}$ at the end of the steel carriage $Y$. The steel carriage is secured to the supporting plate $V$ by a cover plate $Z$ and four screws, the cover plate itself being drilled and recessed on its inner face to receive a second PTFE washer. When secured in position the flat surfaces of the steel carriage $Y$ are at right angles to the central axis of the supporting ring $U$ and the supporting plate $V$ and are in the same plane as the centre of the hole $X$.

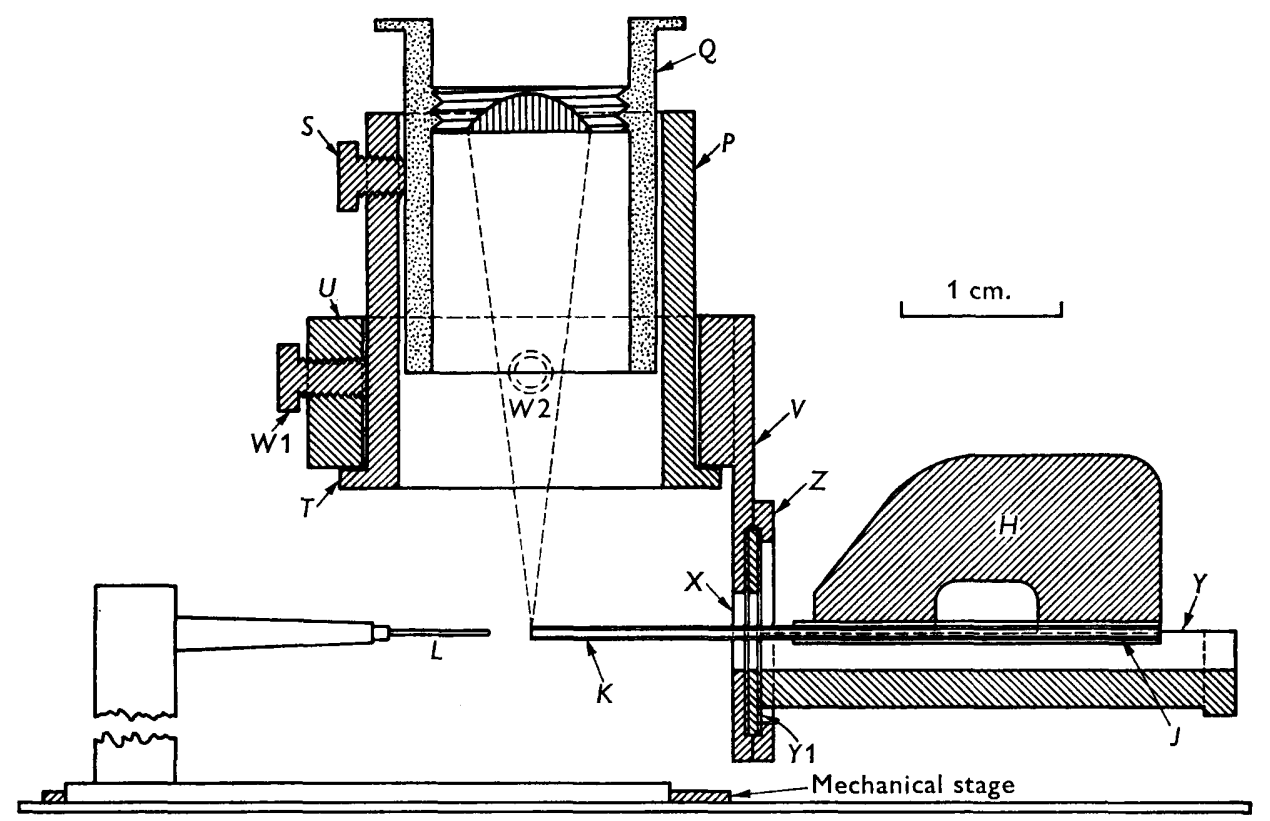

Fig. 8. Schematic drawing of microforge in working position.

(iii) A steel carriage $Y$, machined from a single piece of cylindrical light steel as illustrated, the carriage being a fraction wider than the magnet $H$ used in the manufacture of the magnetic tool-carrier and its length about $12.5 \mathrm{~mm}$. longer than the length of the magnet.

The over-all dimension of the completed microforge tool-carrier must be such that, when attached to the microscope lens, it can be raised or lowered to a position where the horizontal plane through the centre of the hole $X$ in the vertical support $V$ will pass through the focal point of the objective. The diameter of the ring $Y_{I}$ at the end of the steel support $Y$ should be as large as is compatible with the over-all dimensions of the tool in order to achieve maximum stability. When assembled the steel carriage should rotate freely in the support but without any slackness.

The lens used to support the microforge is a $\times 2.5$ lens with a specially attached collar to support the microforge. The author has used a $\times$ Io objective from which the terminal lens had been removed. 
Operation of the microforge. The steel carriage $Y$ is rotated until its flat surface is parallel to the surface of the stage. The magnetic tool-carrier $H+J$, fitted with a glass $\operatorname{rod} K$, is placed on the free end of the steel carriage $Y$ and slipped forwards so that the glass rod passes through the centre of hole $X$ and the end of the magnet comes
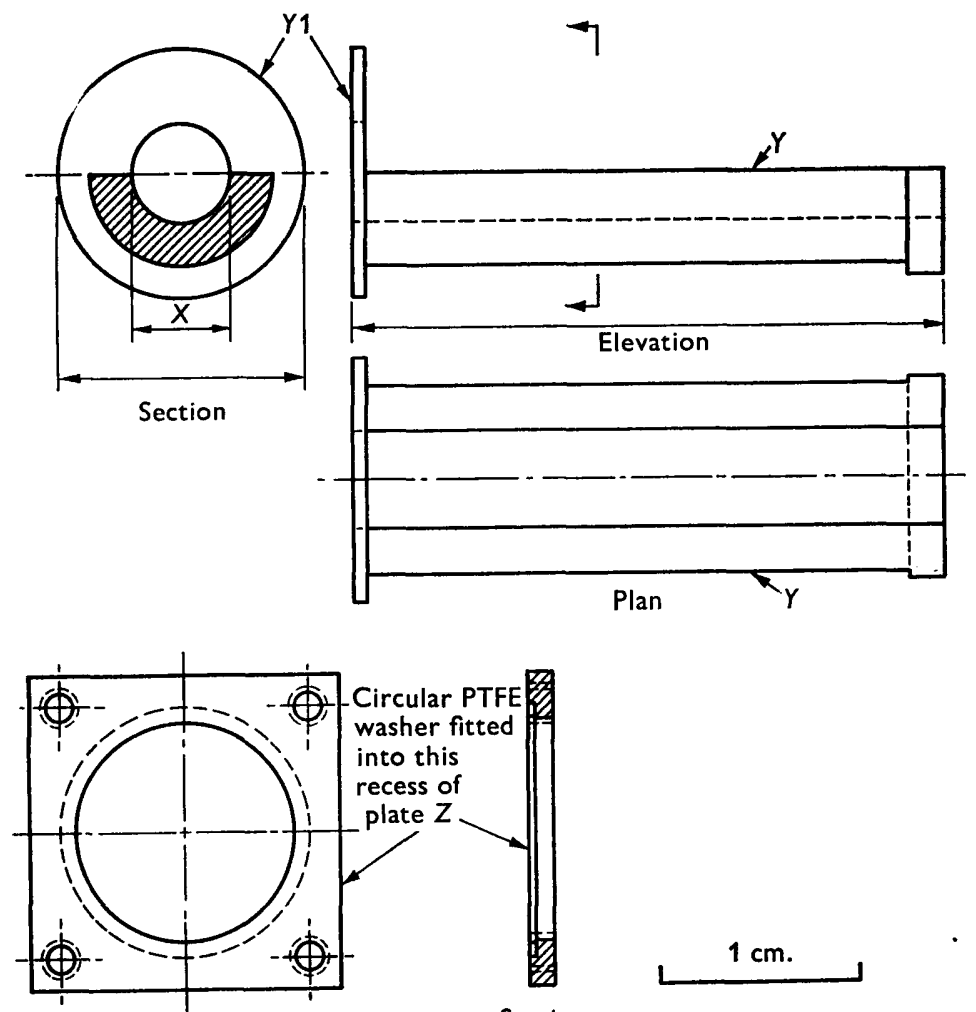

Elevation

Section

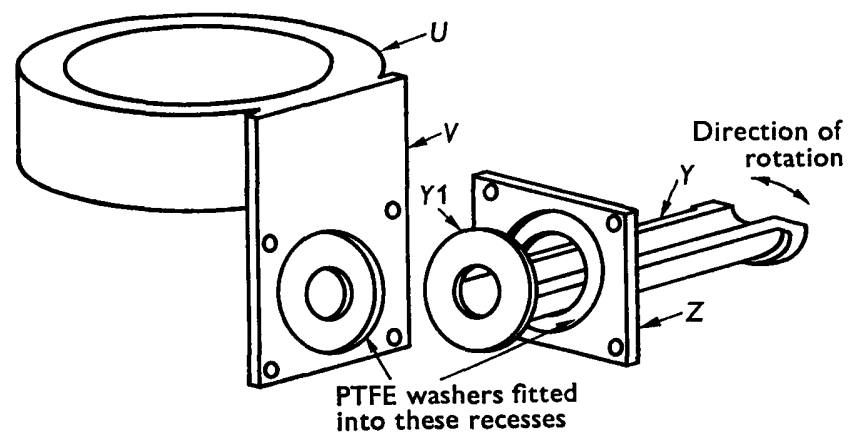

Fig. 9. Microforge.

close to the cover plate $Z$. The height of the collar $P$ is adjusted on the lens casing until the glass rod is brought into focus. The magnet is then retracted until the tip of the glass rod is in the centre of the field; when necessary the focus is readjusted. Provided the glass rod is centrally located it should be possible to rotate the steel carriage $Y$ 
through $90^{\circ}$ either way without the rod going out of focus. Normally some minor adjustment to the magnet will be necessary.

The magnet is then returned to the vertical position and the carriage $Y$ aligned with its long axis parallel to the front end of the microscope stage. Whilst inspecting the instrument from the front of the microscopic stage, the heating stage (Fig. 7) is moved with the mechanical stage until the tip of the heating element $L$ is directly opposite and close to the tip of the glass rod $K$. Then, whilst viewing through the microscope, the height of the barrel of the microscope is adjusted until the platinum/ iridium heating element $L$ is brought to focus in a position opposite to the end of the glass rod. By the application of current to the platinum/iridium wire the glass is melted and drawn out to a fine point by withdrawing the heated element with the mechanical stage.

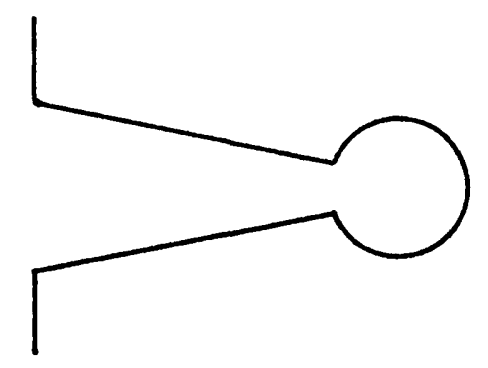

Fig. 10. Shape of platinum wire.

Glass microloops can be manufactured (full details can be obtained from the author by those using the instrument) by the same procedure as that described by de Fonbrun, the temperature of the platinum/iridium wire being controlled with resistance $\boldsymbol{R}_{\mathbf{2}}$. With the apparent closure of the loop the flatness can be checked by rotating the steel carriage $Y$ through an angle of $90^{\circ}$. Any adjustment to the plane of the loop can be made in this position. To bend the loop at the angle required for use on the micromanipulator the plastic screws $W_{1}$ and $W_{2}$ securing the ring $U$ to the collar $P$ are relaxed and the ring rotated so that the steel carriage as a whole passes through the required angle towards the front of the stage. The screw $W_{2}$ is then tightened and the shaft of the loop bent near the base of the loop by gentle pressure with the warm platinum/iridium wire.

To prepare capillaries a procedure similar to that used by de Fonbrun can be used. The microscope is inclined backwards until the barrel is horizontal. It is then anchored on a support which will permit comfortable inspection through the ocular whilst in a sitting position. A glass capillary is fitted into the tool holder and the free end bent into a hook. With the ring $U$ rotated so that the glass rod is in a vertical position, weights can be applied to the hooked end and the glass rod heated from the side in the manner described by de Fonbrun. A refinement of the heating element, which aids in the manufacture of capillaries, is one which permits the holders of the platinum/ iridium wire to rotate through $90^{\circ}$. The platinum wire itself is bent as in Fig. IO. With the loop horizontal to the stage it is positioned so that the centre of the ring is precisely in the centre of the field and in focus with the lens. Allowance must be made for the 
lateral expansion of the heated wire. The loop is then rotated through $90^{\circ}$ and the glass rod pushed down so that it becomes positioned through the centre of the ring. Weights are applied and the element heated. Whilst capillaries can be manufactured quite easily their use in the magnetic tool-carrier presents difficulties which have still to be resolved. The author has found little use for capillaries. Most single-organism isolations can be achieved more quickly by a probe, a probe with a small knob at the end, or a loop.

Once a tool has been prepared the magnet is removed carefully from the forge and applied directly to the slides of the manipulator. Preparation of a new probe, once a probe has been used, simply involves melting the used probe and drawing a new one. The time required is less than that needed to heat and cool a platinum or nichrome wire. The temperature reached ensures sterility. Microloops can be prepared in about two minutes.

\section{DISCUSSION}

The whole apparatus has the advantage of being simple in design, small in size, and adaptable to use on any microscope. The design of the microforge gives threedimensional control over tool manufacture and yields a finished glass tool mounted in the magnetic carriage in which it is subsequently used on the manipulator, thus permitting rapid operation. Used with a low-power phase-contrast lens, singleorganism isolations can quite easily be effected with Bacillus sp. and organisms of similar or greater dimensions. Use of the manipulator with a high-power $(\times 40)$ lens presents problems, not the least of which is condensation of moisture from the agar on the lens surface, for which there is no cure. However, a manipulator of slightly altered design, in which the slides $D$ are fixed a little above the horizontal (angle $\alpha$ in Fig. $I$ is almost 0 ) and the magnet is mounted on top with an undercarriage which ensures that the centre of the tube $J$ is in the same plane as the under surface of the slides $D$, can be used on moderately dry surfaces. Other modifications which would permit manipulation under a cover glass are being considered.

The problem of dust falling on exposed Petri dishes during manipulation has been countered in this laboratory by working with the microscope positioned under a polythene hood with the eyepieces protruding through the wall. The working area is sterilized by means of an ultraviolet light. Clarified agar is used as a matter of routine in any micromanipulative work. 